\title{
The “Off-On" Fluorescence Detection of Alkaline Earth Metal Ions by Using 1-Naphthylacetanilide Derivatives: New Cation Sensors Based on Oligo- ethylene Oxides Connecting 1-Naphthylacetanilide at Its Terminals
}

\author{
Tatsuya Morozumi,* Hiroya Hama,** and Hiroshi NaKamura* \\ *Section of Materials Science, Research Faculty of Environmental Earth Science, Hokkaido University, \\ Sapporo, Hokkaido 060-0810, Japan \\ **Division of Environmental Material Science, Graduate School of Environmental Science, Hokkaido University, \\ Sapporo, Hokkaido 060-0810, Japan
}

\begin{abstract}
New fluorescent ionophores containing the 1-naphthylacetanilide moiety based on oligo-ethylene oxide $\left(\mathbf{1}_{\mathbf{4}}\right.$ and $\left.\mathbf{1}_{\mathbf{5}}\right)$ have been synthesized, and their photochemical behaviors have been studied. In the absence of a metal ion, the 1naphthylacetanilide moiety showed a weak fluorescence emission (fluorescence "off state"). However, complexation with $\mathrm{Ca}^{2+}$ induced a large enhancement effect on the fluorescence intensity of $\mathbf{1}_{\mathbf{4}}$ and $\mathbf{1}_{\mathbf{5}}$ from the naphthalene ring (fluorescence "on" state). This behavior can be explained in terms of a similar twisted intramolecular charge-transfer relaxation mechanism. Such "off-on" fluorescence responses of $\mathbf{1}_{\mathbf{4}}$ and $\mathbf{1}_{\mathbf{5}}$ provide the digital detection of alkaline earth metal ions.
\end{abstract}

(Received April 10, 2006; Accepted April 24, 2006)

Photo-induced electron transfer between a donor and acceptor subsystem in a molecule leads to an intramolecular chargetransfer (ICT) state. The ICT state of the molecule goes down to the ground state through various relaxation pathways. Among of them, in particular, the twisted intramolecular charge transfer (TICT) behavior has been a subject of interest to many photochemical researchers for years. ${ }^{1}$ The TICT model was firstly applied to explain the photophysical property of $p-(N, N-$ dimethylamino)benzonitrile, indicating the dual fluorescence and the emission with a large Stokes shift in a polar solvent., ${ }^{2,3}$ Since then, interpretation by the TICT model has been extended to other structurally related compounds showing anomalous dual fluorescence. Recently, the present authors demonstrated that $N$-phenyl-9-anthracenecarboxamide and $N$-phenyl-1pyrenylcarboxamide derivatives show no fluorescence emission in solution through an ICT relaxation pathway similar to the TICT as the most plausible explanation. On the basis of this observation, we synthesized new fluorescent ionophores containing $N$-phenyl-9-anthracenecarboxamide ${ }^{4}$ and $N$-phenyl1-pyrenylcarboxamide ${ }^{5}$ based on an ethyleneoxy chain, respectively. Upon complexing with metal ions, we could achieve the controlling TICT relaxation pathway with the "offon" fluorescent signal.

These results inspired us to examine a photochemical property of naphthalene amides derivatives. Naphthalene is regarded as a strong tool for a highly sensitive detecting probe in fluoroionophores. ${ }^{6}$ Kawakami et al. studied fluoroionophores containing naphthoic acid derivatives. ${ }^{7-9}$ The naphthyl alkyl amines were used as a photo-induced electron transfer (PET) detection moiety in the field of the molecular recognition chemistry. ${ }^{10,11}$ Although many studies on naphthalene derivatives have been reported, spectroscopic observations about naphthylacetic aromatic amide are rather limited. In this communication, we demonstrate the photochemical behaviors of $\mathbf{1}_{\mathbf{4}}$ and $\mathbf{1}_{\mathbf{5}}$ (Fig. 1) upon the complexing with metal ions by means of fluorescence and NMR spectrometries.

\section{Experimental}

The syntheses of $\mathbf{1}_{\mathbf{4}}$ and $\mathbf{1}_{\mathbf{5}}$ were carried out by the usual technique (Yield: $61.3 \%$ for $\mathbf{1}, 58.3 \%$ for 2 ). ${ }^{12,13}$ Their structures and purities were confirmed by the ${ }^{1} \mathrm{H}-\mathrm{NMR}$ spectra and elemental analyses. Fluorescence spectra were measured by a Shimadzu RF-5300PC at $25^{\circ} \mathrm{C}$. The concentrations of fluorescent reagents were $5 \times 10^{-6} \mathrm{~mol} / \mathrm{dm}^{3}$ in purified acetonitrile. Alkaline earth metal cations were added into the solution of the fluorescent reagent as perchlorate salts.

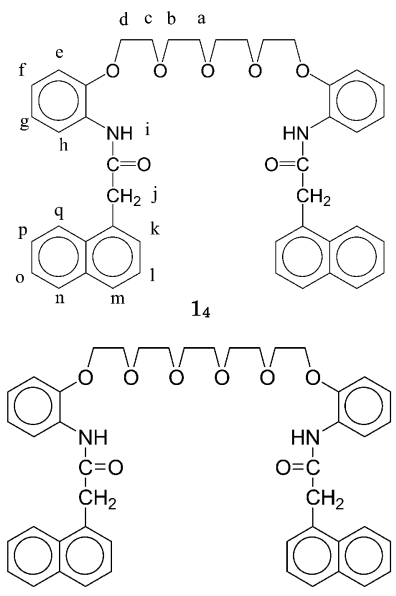

$1_{5}$

Fig. 1 Structures of $\mathbf{1}_{\mathbf{4}}$ and $\mathbf{1}_{\mathbf{5}}$. 

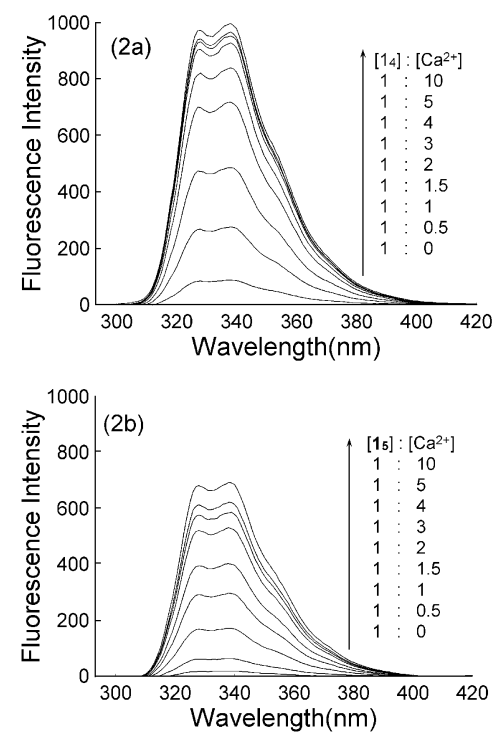

Fig. 2 Fluorescent spectra of $\mathbf{1}_{\mathbf{4}}(\mathrm{a}), \mathbf{1}_{\mathbf{5}}$ (b) and their $\mathrm{Ca}^{2+}$ complex in acetonitrile at $25^{\circ} \mathrm{C}$. Excitation wavelength, $283 \mathrm{~nm}$. [1 $\left.\mathbf{1}_{4}\right]=\left[\mathbf{1}_{5}\right]=$ $5 \times 10^{-6} \mathrm{M}$.

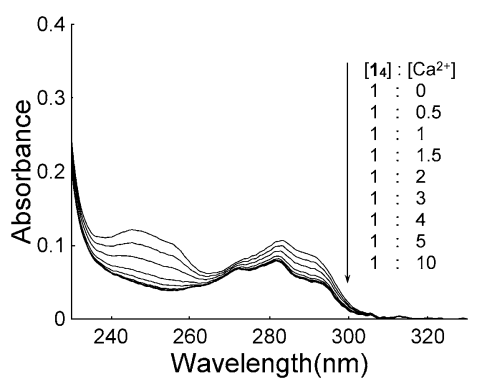

Fig. 3 UV spectra of $\mathbf{1}_{\mathbf{4}}$ and its $\mathrm{Ca}^{2+}$ complex in acetonitrile at $25^{\circ} \mathrm{C}$.

\section{Results and Discussion}

Figure $2 \mathrm{a}$ shows the fluorescence spectra of $\mathbf{1}_{4}$ and its $\mathrm{Ca}^{2+}$ complexes. Fluorescence emissions from the naphthalene moiety in $\mathbf{1}_{\mathbf{4}}$ were quite weak in the absence of metal ions. It can be explained that the quenching of naphthalene fluorescent emission contained two steps, as follows: photo-induced charge transfer occurred between the naphthalene ring and the $\mathrm{N}$ phenyl amide moiety over the methylene bond (naphtharene- $\mathrm{CH}_{2}-\mathrm{CONH}-\mathrm{Ph}$ ). Next, the TICT motion in the $\mathrm{N}$-phenylamide moiety induced a charge-separated state. Thus, $\mathbf{1}_{4}$ showed weak fluorescent emission. On the other hand, the complex formation of $\mathbf{1}_{\mathbf{4}}$ with $\mathrm{Ca}^{2+}$ exhibited naphthalene monomer emission having two maximum $\left(\lambda_{\max }\right)$ at around 345 and $325 \mathrm{~nm}$, and the fluorescence intensity increased upon the addition of $\mathrm{Ca}^{2+}$, respectively. The complexation clearly inhibited the quenching pathway, resulting in a strong fluorescent emission of naphthalene. The excimer of the naphthalene was not observed. This suggested that the benzene ring disturbed an overlapping of naphthalene rings because of their steric hindrance. Upon the addition of other guest ions, such $\mathrm{Sr}^{2+}$ and $\mathrm{Ba}^{2+}, \mathbf{1}_{\mathbf{4}}$ with metal complexes showed a similar "off-on" fluorescence response. Figure $2 \mathrm{~b}$ also shows the fluorescence spectra of $\mathbf{1}_{5}$ and its $\mathrm{Ca}^{2+}$ complexes. Although the increase of the fluorescence intensity was small compared with
Table 1 Complex formation constants $\mathrm{s}^{\mathrm{a}}$ and fluorescent response of $\mathbf{1}_{4}$ and $\mathbf{1}_{5}$ for various metal ions in acetonitrile at $25^{\circ} \mathrm{C}$

\begin{tabular}{ccccc}
\hline \multirow{2}{*}{ Fluoroionophore } & \multicolumn{4}{c}{$\log K\left(I_{\max } / I_{\text {none }}\right)$} \\
\cline { 2 - 5 } & $\mathrm{Mg}^{2+}$ & $\mathrm{Ca}^{2+}$ & $\mathrm{Sr}^{2+}$ & $\mathrm{Ba}^{2+}$ \\
\hline \multirow{2}{*}{$\mathbf{1}_{4}$} & $-{ }^{\mathrm{b}}$ & 5.72 & 5.09 & 4.25 \\
& - & $(11.3)$ & $(10.4)$ & $(5.4)$ \\
$\mathbf{1}_{5}$ & 3.76 & 5.52 & 4.46 & 5.00 \\
& $(2.1)$ & $(25.8)$ & $(16.9)$ & $(27.3)$ \\
\hline
\end{tabular}

a. $K=\left[\right.$ Ligand $\left.\cdot \mathrm{M}^{2+}\right] /[$ Ligand $]\left[\mathrm{M}^{2+}\right]$.

b. Because the changes of fluorescence intensity was small, this value could not be determined.

that of $\mathbf{1}_{\mathbf{4}}$, the fluorescence emission maximum $\left(\lambda_{\max }\right)$ and shape were quite similar to that of $\mathbf{1}_{4} \cdot \mathrm{Ca}^{2+}$. The fluorophore $\mathbf{1}_{5}$ also responded to both of $\mathrm{Sr}^{2+}$ and $\mathrm{Ba}^{2+}$. The fluorescence intensity of free $\mathbf{1}_{5}$ showed a small value $(\sim 50$, at $338 \mathrm{~nm})$ compared with that of free $\mathbf{1}_{\mathbf{4}}(\sim 100$, at $338 \mathrm{~nm})$. The longer oxyethylene chain in $\mathbf{1}_{5}$ had entropical advantages for molecular motion, avoiding the conformation of inhibition for TICT (e.g. pseudo-cyclic comformer).

Figure 3 shows the UV spectra of $\mathbf{1}_{\mathbf{4}}$ and its complexes with metal $\mathrm{Ca}^{2+}$. After the addition of $\mathrm{Ca}^{2+}$, the absorbance of the naphthalene ring slightly decreased, whereas its region did not change. This suggested that fluorescence quenching is not due to a redox-level change for complexation. The absorbance around $270 \mathrm{~nm}$, which could be assigned to the benzene moiety, also decreased. This may reflect the geometry changes along the benzene-NHCO-CH${ }_{2}$-bond axis at the binding event.

The successive increase in monomer emission upon the addition of metal ions will finally cause an inhibition of ICT quenching. The degree of increase of monomer emission clearly depended on the concentrations of metal ions. The fluorescence intensities of $\mathbf{1}_{\mathbf{4}}$ and $\mathbf{1}_{\mathbf{5}}$ at $283 \mathrm{~nm}$ were plotted against the ratio of $\left[\mathrm{M}^{2+}\right] /\left[\mathbf{1}_{\mathbf{4}}\right.$ or $\left.\mathbf{1}_{\mathbf{5}}\right]$. The obtained titration curve clearly indicates the formation of a 1:1 complex with $\mathrm{Ca}^{2+}$. The complex formation constant $(K)$ was determined from the curve by means of a nonlinear least-square curve-fitting method (Marquardt's method), ${ }^{14}$ and listed in Table 1. The fluoroionophores $\mathbf{1}_{4}$ and $\mathbf{1}_{5}$ showed good affinity for $\mathrm{Ca}^{2+}$; the same trend was observed in previous reports.

Fluorescence spectral data evidently showed the structural changes of $\mathbf{1}_{\mathbf{4}}$ and $\mathbf{1}_{5}$ upon complexation with metal ions. To clarify these changes, a ${ }^{1} \mathrm{H}$ NMR study of $\mathbf{1}_{\mathbf{4}}$ was carried out in the absence and presence of $\mathrm{Ca}^{2+}$ in acetonitrile- $d_{3}$ at $30^{\circ} \mathrm{C}$. Peak assignments were made by ${ }^{1} \mathrm{H}^{1}{ }^{1} \mathrm{H}$ COSY and NOESY spectra. In the $\mathbf{1}_{4} \cdot \mathrm{Ca}^{2+}$ complex (refer to the scheme for peak assignments), oxyethylene proton peaks (a, b, c, and d) shifted to low magnetic field $(\Delta \delta=0.03,0.03,0.07$, and $0.67 \mathrm{ppm}$, respectively). The peak of amide protons (i) also showed large lower magnetic field shift changes $(\Delta \delta=0.93 \mathrm{ppm})$. These indicated that $\mathbf{1}_{\mathbf{4}}$ binds to $\mathrm{Ca}^{2+}$ with the ethyleneoxy moiety and carbonyl oxygen in both amide groups cooperatively. However, protons, a, b and c did not show large low-field shifts. This can be considered to mean that low magnetic field shifts induced by complexation were offset by the ring-current effect of the naphthalene ring. Benzene protons (e and $\mathrm{g}$ ) shifted to low magnetic field $(\Delta \delta=0.37$ and $0.26 \mathrm{ppm})$, whereas the proton (f) indicated a high magnetic field shift $(\Delta \delta=-1.15 \mathrm{ppm})$. This can be explained because two of benzenes faced alternatively, and protons (e and g) stood in a deshielding area of the other benzene ring, whereas the proton (f) received the ring-current 


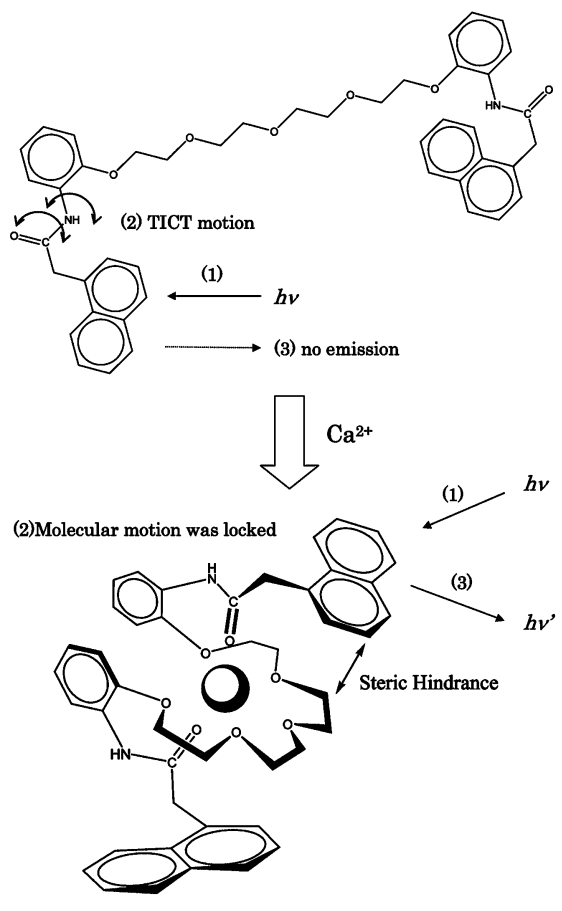

Fig. 4 Schematic presentation of the structural change of $\mathbf{1}_{\mathbf{4}}$ before and after the addition of $\mathrm{Ca}^{2+}$.

effect, resulting in high field shifts. The proton (h) showed an unusual low chemical shift value ( $8.19 \mathrm{ppm})$. To avoid a steric hindrance arising between the carbonyl group and the oxyethylene chain, these two groups must take a trans molecular geometry, and the proton (h) must exist in the deshielding area of the carbonyl group, showing an unusual value. Upon complexing with $\mathrm{Ca}^{2+}$, the geometry changed to a cis form, and the proton (h) came out of this area while showing a normal chemical shift value $(6.48 \mathrm{ppm})$. The chemical shift changes of proton $(\mathrm{j})$ and naphthalene protons $(\mathrm{k}-\mathrm{q})$ could not be observed. $(\Delta \delta=0.0 \mathrm{ppm}$ and $\Delta \delta=0.0--0.19 \mathrm{ppm}$, respectively) These protons did not receive an electronic effect upon complexation.

On the bases of fluorescence and ${ }^{1} \mathrm{H}$ NMR studies, an expected structural change of $\mathbf{1}_{\mathbf{4}}$ before and after the addition of $\mathrm{Ca}^{2+}$ on the ground state is illustrated in Fig. 4. It has been clarified that the carbonyl group and the ethyleneoxy moiety were provided a cooperative strong binding site, resulting in the formation of a pseudo-cyclic comformer. The benzene and oxyethlene moiety induced an inhibition of the rotation around the amide bond in both the ground and excited states, resulting in an inhibition of the TICT relaxation process. The formation of a complex also caused a the steric hindrance between the naphthalene and ethyleneoxy moiety. The photodriven rotation of the naphthalene moiety should be locked by that obstacle. Thus, the fluorescence emission from the naphthalene of $\mathrm{Ca}^{2+} \cdot\left(\mathbf{1}_{\mathbf{4}}\right.$ and $\left.\mathbf{1}_{\mathbf{5}}\right)$ can be observed.

A detailed observation of the relaxation mechanism of naphthylmethyl- $N$-phenyl amide derivatives is now underway. We believe that the present fluorophores $\mathbf{1}_{\mathbf{4}}$ and $\mathbf{1}_{5}$ will be available as a photo-detecting system for analytical use with an "off-on" fluorescent signaling character.

The present authors gratefully acknowledge that financial support for this work was granted by a Grant-in-Aid for Scientific Research (No. 16550065 and 16550064) from the Ministry of Education, Culture, Sports, Science and Technology, Japan.

\section{References and Notes}

1. Z. R. Grabowski and K. Rotkiewicz, Chem. Rev., 2003, 103, 3899.

2. K. Rotkiewicz, K. H. Grellmann, and Z. R. Grabowski, Chem. Phys. Lett., 1973, 19, 315.

3. Z. R. Grabowski, K. Rotkiewicz, A. Siemiarczuk, D. J. Cowley, and W. Baumann, Nouveau J. Chimie, 1979, 3, 443.

4. T. Morozumi, T. Anada, and H. Nakamura, J. Phys. Chem. B, 2001, 105, 2923.

5. T. Morozumi, H. Hiraga, and H. Nakamura, Chem. Lett., 2003, 32, 146.

6. A. P. de Silva, H. Q. N. Gunaratne, T. Gunnlaugsson, A. J. M. Huxely, C. P. McCoy, J. T. Rademacher, and T. E. Rice, Chem. Rev., 1997, 97, 1515, and references therein.

7. J. Kawakami, Y. Komai, T. Sumori, A. Fukushi, K. Shimozaki, and S. Ito, J. Photochem. Photobiol. A, 2001, 139, 71 .

8. J. Kawakami, R. Miyamoto, A. Fukushi, K. Shimozaki, and S. Ito, J. Photochem. Photobiol. A, 2002, 146, 163.

9. J. Kawakami, H. Kimura, M. Nagaki, H. Kitahara, and S. Ito, J. Photochem. Photobiol. A, 2004, 161, 141.

10. T. Gunnlaugsson, B. Bichell, and C. Nolan, Tetrahedron, 2004, 60, 5799.

11. Y. Kubo, M. Kato, Y. Misawa, and S. Tokita, Tetrahedron Lett., 2004, 45, 3769.

12. $N, N^{\prime}$-[Oxybis(3-oxapentamethyleneoxy)-2-phenyl]bis(1naphthylacetamide) (14): Yield $61.3 \%$, oil. ${ }^{1} \mathrm{H}-\mathrm{NMR}$ $\left(\mathrm{CDCl}_{3}\right) \delta=3.27\left(\mathrm{C}-\mathrm{CH}_{2}-\mathrm{O}, \mathrm{t}, 4 \mathrm{H}\right), 3.45\left(-\mathrm{C}-\mathrm{CH}_{2}-\mathrm{O}, \mathrm{t}\right.$, $4 \mathrm{H}), 3.51\left(-\mathrm{C}-\mathrm{CH}_{2}-\mathrm{O}, \mathrm{t}, 4 \mathrm{H}\right), 3.77\left(-\mathrm{C}-\mathrm{CH}_{2}-\mathrm{O}, \mathrm{t}, 4 \mathrm{H}\right), 4.17$ $\left(\mathrm{O}=\mathrm{C}-\mathrm{CH}_{2}-\mathrm{Nap}, \mathrm{s}, \quad 4 \mathrm{H}\right), 6.66$ (aromatic, d, 2H), 6.90 (aromatic, q, 4H), 7.47 - 7.50 (aromatic, m, 8H), 7.80 - 7.88 (aromatic, m, 4H), $7.90(\mathrm{NH}, \mathrm{m}, 2 \mathrm{H}), 8.03$ (aromatic, d, 2H), 8.34 (aromatic, q, 2H). Found: C, 7367; H, 6.46; N, 4.07\%. Calcd. for $\mathrm{C}_{44} \mathrm{H}_{44} \mathrm{O}_{7} \mathrm{~N}_{2}: \mathrm{C}, 74.14 ; \mathrm{H}, 6.22 ; \mathrm{N}$, $3.93 \%$.

13. $N, N^{\prime}$-[Ethylenedioxy-bis(3-oxapentamethyleneoxy)-2phenyl]bis(1-naphthylacetamide) (15): Yield 58.3\%, yellowish oil. ${ }^{1} \mathrm{H}-\mathrm{NMR}\left(\mathrm{CDCl}_{3}\right) \delta=3.27\left(\mathrm{C}-\mathrm{CH}_{2}-\mathrm{O}\right.$, t, $4 \mathrm{H}), 3.46\left(-\mathrm{C}-\mathrm{CH}_{2}-\mathrm{O}, \mathrm{t}, 4 \mathrm{H}\right), 3.54\left(-\mathrm{C}-\mathrm{CH}_{2}-\mathrm{O}, \mathrm{t}, 4 \mathrm{H}\right), 3.59$ $\left(-\mathrm{C}-\mathrm{CH}_{2}-\mathrm{O}, \quad \mathrm{s}, \quad 4 \mathrm{H}\right), \quad 3.78 \quad\left(-\mathrm{C}-\mathrm{CH}_{2}-\mathrm{O}, \quad \mathrm{t}, \quad 4 \mathrm{H}\right), \quad 4.19$ $\left(\mathrm{O}=\mathrm{C}-\mathrm{CH}_{2}-\mathrm{Nap}, \mathrm{s}, 4 \mathrm{H}\right), 6.68$ (aromatic, d, 2H), 6.90 (aromatic, q, 4H), 7.47 - 7.52 (aromatic, m, 8H), 7.80 - 7.88 (aromatic, m, 4H), $7.84(\mathrm{NH}, \mathrm{m}, 2 \mathrm{H}), 8.03$ (aromatic, d, 2H), 8.34 (aromatic, q, 2H). Found: C, 72.83; H, 6.48; N, $3.71 \%$. Calcd. for $\mathrm{C}_{46} \mathrm{H}_{48} \mathrm{O}_{8} \mathrm{~N}_{2}: \mathrm{C}, 73.00 ; \mathrm{H}, 6.39 ; \mathrm{N}$, $3.70 \%$.

14. D. W. Marquardt, J. Soc. Ind. Appl. Math., 1963, 11, 431. 